\title{
Daily Exposure to Sucrose Impairs Subsequent Learning About Food Cues: A Role for Alterations in Ghrelin Signaling and Dopamine D2 Receptors
}

\author{
MJ Sharpe ${ }^{*, 1}$, KJ Clemens', MJ Morris² and RF Westbrook' \\ 'School of Psychology, UNSW, Australia; 'Department of Pharmacology, Medical Sciences, UNSW, Australia
}

\begin{abstract}
The prevalence of hedonic foods and associated advertising slogans has contributed to the rise of the obesity epidemic in the modern world. Research has shown that intake of these foods disrupt dopaminergic systems. It may be that a disruption of these circuits produces aberrant learning about food-cue relationships. We found that rodents given 28 days of intermittent access to sucrose exhibited a deficit in the ability to block learning about a stimulus when it is paired in compound with food and another stimulus that has already been established as predictive of the food outcome. This deficit was characterized by an approach to a cue signaling food delivery that is usually blocked by prior learning, an effect dependent on dopaminergic prediction-error signaling in the midbrain. Administering the $D_{2}$ agonist quinpirole during learning restored blocking in animals with a prior history of sucrose exposure. Further, repeated central infusions of ghrelin produced a deficit in blocking in the same manner as sucrose exposure. We argue that changes in dopaminergic systems resulting from sucrose exposure are mediated by a disruption of ghrelin signaling as rodents come to anticipate delivery of the highly palatable sucrose outside of normal feeding schedules. This suggestion is supported by our finding that both sucrose and ghrelin treatments resulted in increases in amphetamine-induced locomotor responding. Thus, for the first time, we have provided evidence of a potential link between alterations in $D_{2}$ receptors caused by the intake of hedonic foods and aberrant learning about cue-food relationships capable of promoting inappropriate feeding habits. In addition, we have found preliminary evidence to suggest that this is mediated by changes in ghrelin signaling, a finding that should stimulate further research into modulation of ghrelin activity to treat obesity.

Neuropsychopharmacology (2016) 4I, I357-1365; doi:I0.I038/npp.20 I5.287; published online 7 October 2015
\end{abstract}

\section{INTRODUCTION}

Western societies have created an environment where energy-rich, hedonically attractive foods and drinks are abundantly available. Further, advertising campaigns ensure that cues associated with these foods are widespread. The prevalence of such cues promotes overeating and has likely contributed to the recent increased incidence of obesity (Volkow et al, 2011). Overstimulation of mesolimbic systems resulting from excessive intake of hedonic foods may produce dysfunction of reward systems, exacerbating maladaptive feeding behaviors, and adverse health effects. In human studies, obesity is associated with a reduction of $\mathrm{D}_{2 / 3}$ receptor availability and a blunted dopaminergic response to reward, argued to promote overeating (Wang et al, 2001; Van de Giessen et al, 2014; Savage et al, 2014).

Rodent models have shown that highly palatable diets can produce dopaminergic dysfunction. For example, in the intermittent sucrose intake (ISI) model, rodents are given

\footnotetext{
*Correspondence: Dr MJ Sharpe, National Institute on Drug Abuse, 251 Bayview Boulevard, Baltimore, MD 21224, USA, Tel: + |415629|740, E-mail: melissa.sharpe@nih.gov

Received 16 April 2015; revised I September 2015; accepted 4 September 2015; accepted article preview online 14 September 2015
}

restricted access to sucrose at fixed times each day. This model produces changes in neural circuitry that are similar to those that result from drugs of abuse, such as amphetamine (Avena et al, 2008; Furlong et al, 2014). Specifically, daily exposure to sucrose repeatedly releases dopamine in the nucleus accumbens $(\mathrm{NaCC})$, decreases $\mathrm{D}_{2}$ receptor binding, and increases $D_{1}$ receptor binding in the NaCC (Avena et al, 2008). As noted, obese humans also exhibit a decrease in $\mathrm{D}_{2 / 3}$ receptor availability, and rodents sensitized to amphetamine, like those subjected to the ISI protocol, show this dissociation between $\mathrm{D}_{1}$ and $\mathrm{D}_{2}$ receptors (Wang et al, 2001; Nelson and Killcross, 2013; Furlong et al, 2014).

Contrasting effects on $\mathrm{D}_{1}$ and $\mathrm{D}_{2}$ receptors following repeated daily restricted exposure to sucrose are not observed in rodents given continuous access to sucrose (Avena et al, 2008; Furlong et al, 2014). A disruption of ghrelin signaling may underlie this difference. Ghrelin is an orexigenic hormone, which stimulates feeding at scheduled meal times; indeed, its release tracks changes in meal times (Le Sauter et al, 2009). The ghrelin receptor (GHS-R1a) is abundant in mesolimbic circuitry and ghrelin is thought to have a role in the ingestion elicited by hedonically attractive foods (Abizaid et al, 2006; Schellekens et al 2013). In line with this, lateralventricular ghrelin infusion increases dopaminergic firing 
in the ventral tegmental area (VTA) and intra-VTA infusion of ghrelin enhances preference for palatable foods over standard chow (Egecioglu et al, 2010; Cone et al, 2014). Thus, the release of ghrelin as rodents come to anticipate sucrose access at regular times outside feeding schedules may disrupt dopaminergic systems.

Evidence that ISI produces changes in dopaminergic systems raises the possibility that such intake will affect subsequent learning about cue-food relationships. The blocking effect has been used to show that such learning is regulated by the firing of midbrain dopamine neurons (Schultz et al, 1997). A protocol to produce the blocking effect consists of exposing subjects to pairings of a cue (eg, a light) with food in stage one. This is followed by pairings of a compound composed of the light and a second cue (eg, a noise) with food in stage two. At test, the noise elicits less conditioned responding than a noise trained in compound with the light from the outset. Prior training with the light is said to have blocked learning about the noise. This phenomenon is dependent on prediction-error mechanisms, specifically, the release of dopamine in the VTA and NaCC when a motivationally significant outcome is unexpectedly delivered as a consequence of cue presentation (and the absence of this signal when the outcome is fully predicted in stage two, as is the case in blocking; Schultz et al, 1997; Day et al, 2007). Thus, in view of evidence that ISI results in changes in dopamine systems, we hypothesized that ISI would alter prediction-error learning via changes in ghrelin signaling. If true, this alteration in prediction-error learning could lead to aberrant food-cue associations, which in turn could lead to maladaptive feeding behaviors.

We tested these hypotheses by first examining whether ISI produced a deficit in the blocking phenomenon. We then investigated whether the effect on blocking was dependent on $\mathrm{D}_{2}$ receptors, known to be disrupted in people with obesity (Wang et al, 2001). Rats with a history of ISI received the $\mathrm{D}_{2}$ agonist quinpriole during the compound phase of the blocking to assess whether stimulation of $\mathrm{D}_{2}$ receptors alleviated the blocking deficit induced by ISI. We next examined whether repeated lateral-ventricular ghrelin infusions produced a blocking deficit in the same manner as ISI. Finally, we assessed whether pretreatment with sucrose or ghrelin altered locomotor response to amphetamine challenge, where an increase in amphetamine-induced locomotor activity would indicate a change in dopaminergic receptors in the VTA and NaCC (Kalivas and Stewart, 1991).

\section{MATERIALS AND METHODS}

\section{Subjects}

Subjects were experimentally naive male Sprague-Dawley rats (Animal Resource Centre, Australia), 10-14 weeks old. Rats were housed eight per cage $(26 \times 59 \times 37 \mathrm{~cm})$, in a temperature- and humidity-controlled environment $\left(22^{\circ} \mathrm{C}\right)$ on a 12-h-light/dark cycle (on 0700 hours). Procedures took place during the light cycle. Experiments were approved by the University of New South Wales Animals Care and Ethics Committee.

\section{Apparatus}

Blocking. Procedures took place in eight standard chambers $(30 \times 24 \times 22 \mathrm{~cm}$; Med Associates, VT) individually housed in light- and sound-attenuating compartments. Each chamber was equipped with a pellet dispenser that delivered pellets into magazine. Magazine entry was detected by infrared sensors mounted across the magazine. Two panel lights were located on the right-hand wall of the chamber above the magazine. A 3-W-house light was located on the upper left-hand wall of the chambers. Each chamber contained a white noise generator and a heavy duty relay that delivered a $5-\mathrm{kHz}$ clicker stimulus. A computer equipped with MED-PC software (Med Associates, VT) controlled experimental events and recorded responses.

Locomotor assessment. Sixteen chambers $(30 \times 30 \times 24 \mathrm{~cm}$; Med Associates, VT) were used. Each consisted of aluminum side panels, Plexiglas front and back panels, and a stainless steel rod floor. Locomotor activity was measured by four infrared photobeams positioned on each sidewall $(1.5 \mathrm{~cm}$ above floor). Each chamber was housed in individual sound-attenuating boxes with fans providing masking noise. All data were recorded by MED-PC software (Med Associates, VT).

\section{Diet Manipulations}

The sucrose protocol was adapted from Kendig et al (2013). Rats were maintained on ad libitum chow and water. Between 1500 and 1700 hours each day for 28 days, rats were given access to a $10 \%$ sucrose solution (sucrose group; CSR white sugar, Australia), equivalent to commercially available soft drinks. In experiment 1 , we assessed the impact of a saccharin solution on the blocking effect in a separate group to control for the possibility that access to a sweet solution might alter the palatability of food reward used in our procedures. This group received a $0.1 \%$ saccharin solution (Sigma-Aldrich, Australia); a concentration selected because it is equivalent in preference to the $10 \%$ sucrose in choice tests (Kendig et al 2013). Solutions were provided in home cages with four bottles per cage to ensure rats could freely consume. Chow and water were removed during this 2 - $h$ period for all groups.

\section{Drugs}

Drugs were dissolved in $0.9 \%$ saline. Quinpirole (QUI, Sigma-Aldrich; Australia) was injected subcutaneously at a dose of $0.1 \mathrm{mg} / \mathrm{kg} 15 \mathrm{~min}$ before stage-two blocking sessions. $D$-amphetamine sulfate (National Measurement Institute; Australia) was injected intraperitoneally at either 0.5 or $1.0 \mathrm{mg} / \mathrm{kg}$.

\section{Central Ghrelin Administration}

Rats underwent surgery to implant cannulae targeting the lateral ventricles. The surgical procedure is described elsewhere (Sharpe and Killcross, 2015). In brief, unilateral stainless steel guide cannulae (28 Gauge; Plastics One, VA) were lowered $0.5 \mathrm{~mm}$ dorsal to the infusion site (co-ordinates relative to bregma; anteroposterior, -1.0 ; mediolateral, +1.5 ; 
dorsoventral, -4.2). Active rat ghrelin ( $1 \mu \mathrm{g}$ in $1 \mu \mathrm{l} 0.9 \%$ saline, $301.67 \mu \mathrm{M}$; Polypeptide laboratories, Switzerland) or $0.9 \%$ saline was infused at $0.2 \mu \mathrm{l} / \mathrm{min}$ by inserting a 33 -Gauge internal cannula into the guides. After 7 days of recovery, rats underwent 14 days of infusions at the same time each day. Internal cannulae were connected to a $25-\mu \mathrm{l}$ glass syringe (Hamilton Syringes, NV) attached to an infusion pump (World Precision Instruments, FL) projecting an additional $0.5 \mathrm{~mm}$ from the guide cannula. This amount of ghrelin was chosen as it has been found to elicit dopaminergic spiking in the $\mathrm{NaCC}$ at the time of infusion (Cone et al, 2014) with ghrelin-related effects lasting for up to $2 \mathrm{~h}$ (Jerlhag, 2008). Rats were culled with sodium pentobarbital (Virbac, Sydney, Australia) and decapitated. Brains were removed, placed on a Peltier element of a cryostat (Leica-microsystems, Sydney, Australia), and frozen overnight. Coronal sections (40 microns) were cut through the lateral ventricles and mounted. Tissue was stained using $1 \%$ cresyl-violet Nissl stain and assessed for placement of cannulae microscopically. Rats with cannulae placements outside of the ventricles, as defined by Paxinos and Watson (1998), were excluded from statistical analyses.

\section{Behavioral Procedures}

Blocking. All conditioned stimuli were $10 \mathrm{~s}$ in duration, separated by a variable inter-trial interval (mean $=2 \mathrm{~min}$ ). Four stimuli were used (click, noise, flashing panel lights, and the house light; Table 1). Physical identity of stimuli was counterbalanced within modality. Blocking experiments were conducted in darkness. Before conditioning, rats received two 30-min sessions of magazine training with pellets delivered according to a 60-s random interval schedule. The unconditioned stimulus was a $40-\mathrm{mg}$ grain pellet (dustless precision grain-based pellets, Bio-Serv, NJ).

Stage one. Rats received 12 conditioning sessions, involving 14 presentations of a 10-s visual stimulus (panel lights

Table I Experimental Design for Experiments I, 2, and 3

\section{Blocking procedure}

\begin{tabular}{lllll}
\hline Exp & Pretreatment/drug & Stage one & Stage two & Test \\
\hline I & Sucrose, saccharin, or water & $\mathrm{A}+$ & $\mathrm{AB}+\mathrm{CD}+$ & $\mathrm{B}$ D \\
2 & Sucrose or water/quinpirole or saline & $\mathrm{A}+$ & $\mathbf{A B}+\mathbf{C D}+$ & $\mathrm{B}$ D \\
3 & Ghrelin or saline & $\mathrm{A}+$ & $\mathrm{AB}+\mathrm{CD}+$ & $\mathrm{B}$ D \\
\hline
\end{tabular}

Pretreatment indicates the diet or neuronal manipulation taken place before the behavioral procedure. That is, rats received sucrose or ghrelin treatment before commencing the blocking procedures and so any effect on learning is due to a long-term change in learning produced by a history of sucrose or ghrelin exposure. The drug column indicates the drug animals received during stage-two blocking where CSA fails to block CSB (marked in bold). During stage-one conditioning, rats were initially trained with visual stimulus $(A)$ paired with reinforcement. In stage two, rats were presented with two audio-visual compounds, one comprising the previously trained stimulus (AB) and another novel compound (CD). At test, animals were presented with stimuli $B$ and $D$ alone under extinction. All stimuli were counterbalanced within modality where stimulus $A$ and $C$ are visual stimuli (either flashing panel lights or a house light) and stimuli B and D are auditory stimuli (either clicker or a white noise).

+ indicates delivery of a 40-mg grain pellet. or house light; counterbalanced; labeled A), which terminated in pellet delivery. In the last two sessions, rats also received two non-reinforced presentations of the other visual stimulus (labeled C) to facilitate discrimination between the two visual stimuli without giving enough exposure to impair subsequent learning about this stimulus (Rhodes and Killcross, 2004; Sharpe and Killcross, 2014).

Stage two. Here two compound stimuli were formed. One comprised stimulus A and a novel auditory stimulus (labeled B: click or noise), and the other comprised stimulus $\mathrm{C}$ and another novel auditory stimulus (labeled D: noise or click). Rats received six compound training sessions, each consisting of six $\mathrm{AB}$ reinforced trials and six reinforced $\mathrm{CD}$ trials. Each compound presentation co-terminated with the presentation of a food pellet.

Test. Auditory stimuli (B and D) were each presented alone without reinforcement. The order of stimulus presentation was fully counterbalanced. The session contained 12 trials, six presentations of each stimulus. Rats were given two test sessions, using the opposite counterbalancing for each test (eg, rats given B on the first trial in test 1 were given $\mathrm{D}$ on the first trial in test 2). Analyses were conducted on responding over the first four trials of these tests as responding extinguished quickly after the first few trials as is commonly observed in the literature (Baxter et al, 1999; Iordanova et al, 2006; Sharpe and Killcross, 2014).

\section{Locomotor Assessment}

Locomotor assessment took place across 5 days, 30 days following sucrose or ghrelin treatments. On day 1, rats were exposed to the locomotor chamber for $1 \mathrm{~h}$ in order to familiarize them with this environment. On day 2, rats were placed in the chambers for $30 \mathrm{~min}$, given a saline injection and then placed back in the chambers for $60 \mathrm{~min}$. On each of days $3-5$, rats received an injection of saline, $0.5 \mathrm{mg} / \mathrm{kg}$, and $1 \mathrm{mg} / \mathrm{kg}$ amphetamine, counterbalanced according to a Latin square design.

\section{Statistics}

Data were analyzed with one-way or mixed-design repeatedmeasures ANOVA, where analyses of simple main effects were used to clarify significant interactions. Rats whose magazine entries were two SDs from the group mean were excluded from all analyses (Knox et al, 2012; Andrews et al, 2012). Using this criterion, we excluded four rats from the total $n=84$, but no more than one rat was excluded from a treatment group in any instance. A further three rats were removed from all analyses due to sickness or dislodgement of cannulae in experiments 3 and $4 \mathrm{~b}$.

\section{RESULTS}

\section{Experiment 1: ISI Abolishes the Blocking Effect}

Sucrose and saccharin intakes for all experiments are presented in the Supplementary Procedures. We found that rats increased their consumption of sucrose across the 28 days of the procedure. Despite this, there was no 


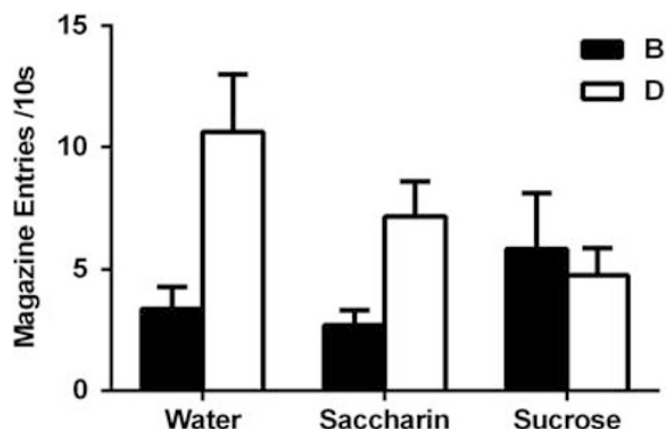

Figure I Intermittent access to sucrose abolishes the blocking effect. Rats given access to water $(n=8)$ or saccharin $(n=7)$ before the blocking procedure exhibited higher levels of responding towards stimulus $D$ relative to stimulus $\mathrm{B}$, demonstrating the blocking effect. In contrast, animals given intermittent access to sucrose $(n=8)$ before the blocking procedure failed to demonstrate this effect, responding equally to both stimuli $B$ and $D$.

difference in body weights between the groups at the end of the procedure because rats in the sucrose group reduced their intake of chow. The sucrose and saccharin solutions are equally preferred in choice tests but rats drank more of the sucrose than the saccharin, presumably because of the caloric contents of the former. Given this difference in intake, future studies should control intakes by providing rats in the ISI protocol with an amount of sucrose equal to the amount of saccharin consumed by rats in saccharin-treated groups. There was no between-group difference in acquisition of the response to CSA in stage one or to the $\mathrm{AB}$ and $\mathrm{CD}$ compounds in stage two (Supplementary Information). The critical test results are shown in Figure 1. This figure suggests that rats in the saccharin and water groups exhibited higher levels of responding to $\mathrm{D}$ than to $\mathrm{B}$, showing that the pretrained A blocked conditioning of $\mathrm{B}$ relative to the effect of $\mathrm{C}$ on D. In contrast, there was little difference between responding to $B$ and $D$ in the sucrose group, suggesting that A had failed to block B. A mixed-design repeated-measures ANOVA revealed a main effect of stimulus (ie, B $v s$ D; $\left.\mathrm{F}_{(1,20)}=8.72, P<0.05\right)$, and a significant between-group interaction $\left(\mathrm{F}_{(2,20)}=4.46, P<0.05\right)$. Analyses of simple main effects demonstrated that the source of this interaction was a significant difference between responding to $\mathrm{B}$ and $\mathrm{D}$ in the water group $\left(\mathrm{F}_{(1,20)}=12.94, P<0.05\right)$, a non-significant trend towards a blocking effect in the saccharin-treated group $\left(\mathrm{F}_{(1,20)}=4.22, \quad P=0.053\right)$, and no effect in the sucrose-treated group $(F<1)$. In addition, analyses of between-subjects simple main effects demonstrated that responding to $\mathrm{D}$ differed between sucrose- and watertreated animals $\left(\mathrm{F}_{(1,20)}=5.77, P<0.05\right)$, but not between saccharin- and water-treated animals $\left(\mathrm{F}_{(1,20)}=1.89\right.$, $P>0.05)$, or saccharin- and sucrose-treated animals $(\mathrm{F}<1)$. This suggests that the sucrose-treated animals learnt less about D. There was no overall difference in levels of responding between-groups during CS presentations on test $(\mathrm{F}<1)$. Further, there was no between-group difference in responding during the $10 \mathrm{~s}$ pre-CS period $\left(\mathrm{F}_{(2,22)}=1.76\right.$, $P>0.05)$. These data demonstrate that ISI abolishes the blocking effect and suggest that ISI also affected what was learned about the elements of the control compound. A specific deficit in the blocking effect was supported by our additional finding that another group of animals given ISI

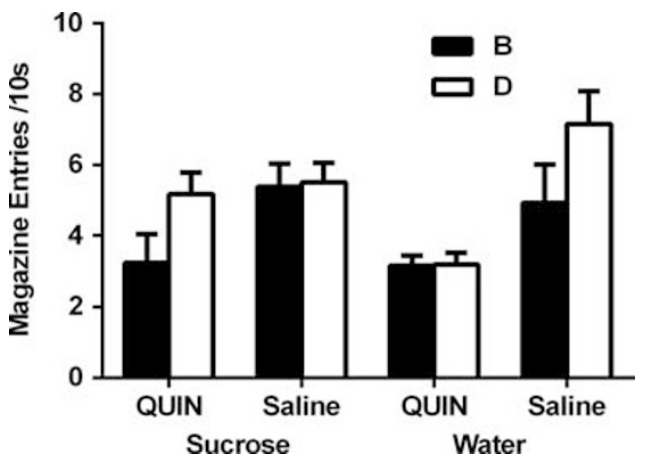

Figure 2 D2 agonist quinpirole recovers the deficit in blocking exhibited by animals given intermittent access to sucrose. Animals in the sucroseQUIN group $(n=7)$ exhibit the blocking effect, similar to the water-saline group $(n=6)$. Again, animals in the sucrose-saline $(n=7)$ failed to demonstrate the blocking effect replicating our basic effect. This is consistent with the notion that a deficit in the blocking effect is mediated by a downregulation of $D_{2}$ receptors. Animals in the water-QUIN group $(n=5)$ also failed to demonstrate the blocking effect, suggesting that $D_{2}$ receptors regulate the blocking effect under normal circumstances.

did not demonstrate a deficit in a Pavlovian-to-instrumental transfer effect with the auditory B and D cues, indicating that the blocking deficit was not due to an inability of rats given ISI to discriminate between these cues (Supplementary Figure 1).

\section{Experiment 2: The $\mathrm{D}_{2}$ Agonist, Quinpirole, Restores the Blocking Effect in Animals Given ISI}

We examined whether the $\mathrm{D}_{2}$ agonist, QUI, restored the blocking effect in rats with a history of ISI. Rats received daily access to sucrose (or water) in the manner described. Following stage-one conditioning where rats received pairings of A and the pellet US, rats in each of the two groups were injected with either QUIN or saline before each stagetwo conditioning session where the $\mathrm{AB}$ and $\mathrm{CD}$ compounds were each paired with the pellet US. QUIN was administered during stage two to assess its effects on the blocking of $B$ by the pretrained A. Finally, rats were tested drug free with B and $\mathrm{D}$ as described. There was no between-group difference in acquisition of the conditioned response to A during stage one or in responding to the $\mathrm{AB}$ and $\mathrm{CD}$ compounds across their pairings with the pellet US during stage two (although QUIN slightly increased pre-CS responding; see Supplementary Information). Figure 2 shows the test data. Water-treated rats responded more to $\mathrm{D}$ than $\mathrm{B}$, indicating blocking of $B$, whereas sucrose-treated rats responded just as much to D as B, replicating the failure of blocking observed in the previous experiment. Critically, rats in group sucroseQUIN responded more to $\mathrm{D}$ than $\mathrm{B}$, indicating that QUI reinstated the blocking effect in sucrose-treated rats, whereas rats in group water-QUIN responded just as much to D as B, indicating that QUI had abolished the blocking effect in the water-treated rats. A mixed-design repeated-measures ANOVA revealed a main effect of stimulus (ie, B $v s$ D; $\left.\mathrm{F}_{(1,21)}=5.94, P<0.05\right)$ and a significant three-way interaction between-diet group, drug, and stimulus $\left(\mathrm{F}_{(1,21)}=5.13\right.$, $P<0.05)$, suggesting that the effect of drug depended on the diet group. Simple main effects demonstrated that the source of this interaction was due to significant blocking in the 
sucrose-QUIN $\left(\mathrm{F}_{(1,21)}=5.37, P<0.05\right)$ and the water-saline group $\left(\mathrm{F}_{(1,21)}=6.27, P<0.05\right)$, but the absence of blocking in the sucrose-saline and water-QUIN groups (Fs $<1)$. Analyses of the between-subjects simple main effects showed that there was no statistically significant difference in responding to $\mathrm{D}$ between sucrose-saline and water-saline groups was not significant $\left(\mathrm{F}_{(1,21)}=3.21, P>0.05\right)$ or between sucroseQUIN and sucrose-saline groups $(F<1)$. However, there was a non-significant trend towards a difference in responding to $B$ between sucrose-QUIN and sucrose-saline groups $\left(F_{(1,21)}=4.11, P=0.055\right)$. These data suggest the blocking deficit in sucrose-treated rats was alleviated because QUI had restored the ability of $A$ to reduce learning about $B$. In addition, there was a between-subject difference in responding to the CSs during the extinction test in rats given QUI relative to saline (ie, sucrose-QUIN and water-QUIN; $\left.\mathrm{F}_{(1,21)}=12.12, P<0.05\right)$, suggesting that the $\mathrm{D}_{2}$ agonist QUI resulted in a general reduction in learning about the CSs during stage-two or rendered what had been learned in that stage less resistant to extinction relative to saline. There was no between-group difference in levels of responding during the 10 -s pre-CS period $\left(\mathrm{F}_{(3,21)}=1.19, P>0.05\right)$. These data confirm that the history of sucrose attenuates blocking (group sucrose-saline) and showed that the $\mathrm{D}_{2}$ agonist restored blocking in rats with a history of sucrose (group sucrose-QUIN). Further, the absence of blocking in the water-QUIN group suggests that $\mathrm{D}_{2}$ receptors are involved in the blocking phenomenon under normal circumstances. Taken together, these results suggest that ISI attenuates the blocking effect through alterations of $\mathrm{D}_{2}$ receptors.

\section{Experiment 3: Repeated Ghrelin Infusions Abolish the Blocking Effect}

Experiment 3 examined whether central ghrelin infusions, such as ISI, also produced the deficit in blocking. There was no between-group difference in the rate of acquisition to $\mathrm{A}$ across stage one or to the $\mathrm{AB}$ and $\mathrm{CD}$ compounds across stage two in rats with a history of ghrelin infusions (Supplementary Information). Figure 3 shows the test results. Rats in the saline group exhibited higher levels of responding to $\mathrm{D}$ than $\mathrm{B}$, confirming blocking of $\mathrm{B}$ by the pretrained $\mathrm{A}$ relative to the effect of $C$ on $D$. In contrast, rats in the ghrelin group failed to exhibit this difference. A mixed-design repeated-measures ANOVA demonstrated that there was a significant main effect of stimulus $\left(\mathrm{F}_{(1,11)}=6.36, P<0.05\right)$, with a significant between-group interaction $\left(F_{(1,11)}=5.78\right.$, $P<0.05)$. There was no between-group difference in levels of responding during the test $\left(\mathrm{F}_{(1,11)}=1.50, \quad P>0.05\right)$ or in levels of responding during the pre-CS period $\left(\mathrm{F}_{(1,11)}=1.23, P<0.05\right)$. These results demonstrate that a history of chronic ghrelin infusions, such as ISI, disrupts the blocking effect.

\section{Experiment 4a: ISI Increases Amphetamine-Induced Locomotor Activity}

We used a subset of rats from experiment 2 (sucrose $n=8$, water $n=8$; equally derived from QUI and saline groups) and measured their locomotor response to amphetamine. Each rat was injected with saline, $0.5 \mathrm{mg} / \mathrm{kg}$, and $1.0 \mathrm{mg} / \mathrm{kg}$ of amphetamine. Figure $4 \mathrm{a}$ shows the average levels of post-

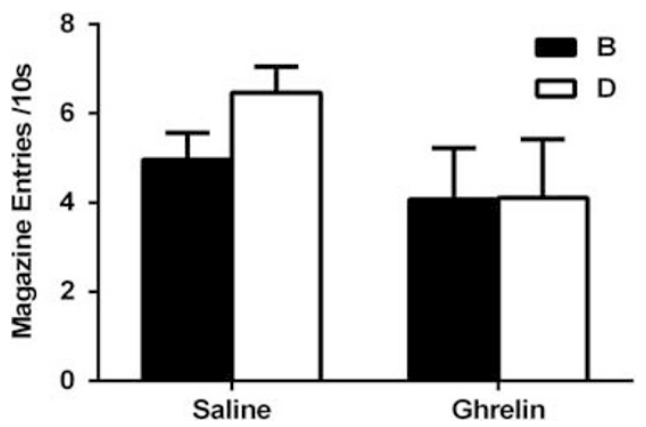

Figure 3 Chronic infusion of ghrelin abolishes the blocking effect. Rats in the saline group $(n=6)$ exhibited higher levels of responding to $D$ relative to $B$, exhibiting the blocking effect. In contrast, animals in the ghrelin group $(n=7)$ exhibited similar levels of responding across both stimuli, failing to demonstrate the effect.
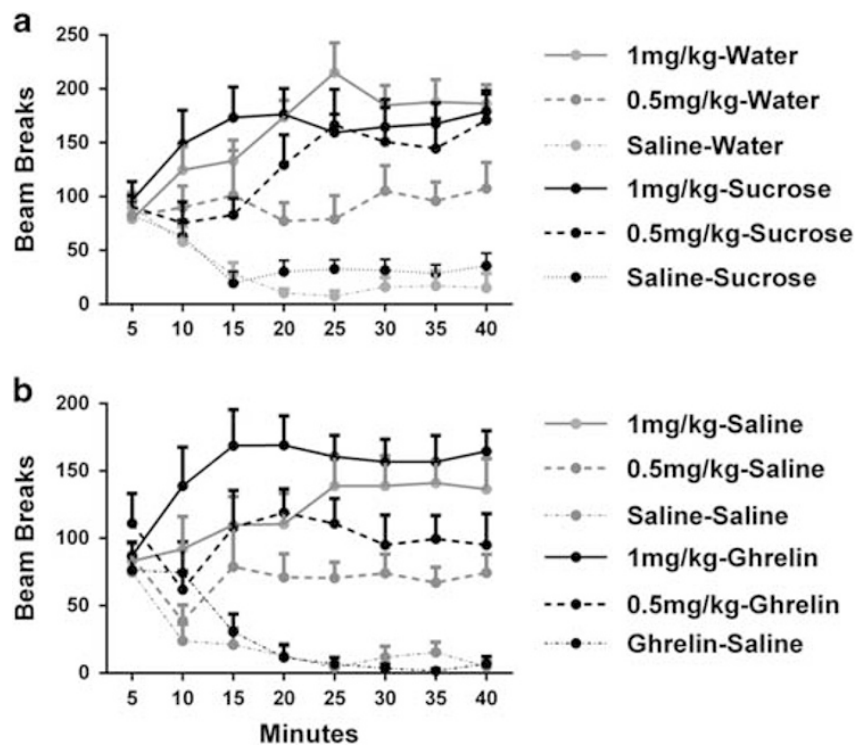

Figure 4 Both sucrose and ghrelin treatments increase locomotor activity in response to amphetamine challenge. (a) Impact of intermittent access to sucrose on locomotor response to amphetamine challenge. Prior treatment with sucrose $(n=8)$ led to a greater persistence of locomotor activity following low $0.5 \mathrm{mg} / \mathrm{kg}$ of amphetamine relative to the water group $(n=8)$. (b) Impact of prior infusions of ghrelin $(n=8)$ on locomotor response to amphetamine challenge. Ghrelin treatment resulted in a greater locomotor response to both the low $0.5 \mathrm{mg} / \mathrm{kg}$ and high I $\mathrm{mg} / \mathrm{kg}$ dose of amphetamine relative to saline treatment $(n=8)$.

injection locomotor responding for the $40 \mathrm{~min}$ following injection across 5-min time bins for rats with an without a history of sucrose exposure. The figures shows that rats in the sucrose group exhibited higher locomotor responding at the low dose $(0.5 \mathrm{mg} / \mathrm{kg})$ relative to the water control group, whereas both groups exhibited the same high response to the high dose $(1 \mathrm{mg} / \mathrm{kg})$ of amphetamine. This was confirmed by statistical analyses, conducted on locomotor activity across the eight 5-min time bins. A mixed-design repeatedmeasures ANOVA of the data from the three doses (ie, saline, $0.5 \mathrm{mg} / \mathrm{kg}$, and $1 \mathrm{mg} / \mathrm{kg}$ ) across time revealed a main effect of dose $\left(\mathrm{F}_{(2,28)}=61.28, P<0.05\right)$, with no dose $x$ group interaction $\left(\mathrm{F}_{(2,28)}=1.36, P>0.05\right)$, suggesting activity 
was directly related to dose. There was a main effect of time $\left(\mathrm{F}_{(7,98)}=3.87, \quad P<0.05\right)$, and no time $x$ group interaction $(\mathrm{F}<1)$, demonstrating that locomotor activity decreased across the session at the same rate across groups. There was a significant dose $x$ time interaction $\left(\mathrm{F}_{(14,196)}=\right.$ $7.17, P<0.05)$, showing higher doses elicited more persistent activity. Critically, there was a significant three-way interaction between dose, time, and group $\left(\mathrm{F}_{(14,196)}=2.41\right.$, $P<0.05)$. Analysis of simple main effects revealed this was due to a significant increase in the persistence of locomotor activity at the low dose of amphetamine in the sucrose group $\left(\mathrm{F}_{(1,14)}=4.89, P<0.05\right)$.

\section{Experiment 4b: Ghrelin Infusions Increase Amphetamine-Induced Locomotor Activity}

We used experimentally naive rats to assess the effects of repeated ghrelin infusions 30 days before assessment of the amphetamine-induced locomotor activity. Figure $4 \mathrm{~b}$ shows the mean levels of activity across 5-min time bins following injection of saline, $0.5 \mathrm{mg} / \mathrm{kg}$, and $1 \mathrm{mg} / \mathrm{kg}$ of amphetamine in rats with and without a prior history of ghrelin infusions. Inspection suggests that rats in the ghrelin group exhibited higher locomotor responding to both doses of amphetamine than the control rats, but the same low response to the saline injection. This was confirmed by statistical analyses. Analyses were conducted on locomotor activity across eight 5-min bins. A mixed-design repeated-measures ANOVA of the data from the three doses (ie, saline, $0.5 \mathrm{mg} / \mathrm{kg}$, and $1 \mathrm{mg} / \mathrm{kg}$ ) across time revealed a main effect of dose $\left(\mathrm{F}_{(2,28)}=127.27, \quad P<0.05\right)$, with no interaction by group $\left(\mathrm{F}_{(2,28)}=2.32, \quad P>0.05\right)$, suggesting activity was directly related to dose. There was a main effect of time $\left(F_{(7,98)}=\right.$ $10.38, P<0.05)$ and a significant time $x$ group interaction $\left(\mathrm{F}_{(7,98)}=13.28, P<0.05\right)$, showing rats in the ghrelin group exhibited more persistent activity to amphetamine. There was a dose $x$ time interaction $\left(\mathrm{F}_{(14,196)}=8.84, P<0.05\right)$, and a three-way interaction $\left(\mathrm{F}_{(14,196)}=3.81, P<0.05\right)$. Simple main effects analyses revealed the source of this interaction was a persistence of the between-group difference in activity elicited by both low $\left(\mathrm{F}_{(1,14)}=14.25, P<0.05\right)$ and higher doses of amphetamine $\left(\mathrm{F}_{(1,14)}=6.69, P<0.05\right)$, but no differences in activity elicited by saline $(\mathrm{F}<1)$. There was a significant between-group difference in overall activity levels, showing that rats given ghrelin infusions demonstrated higher activity in response to amphetamine injections $\left(\mathrm{F}_{(1,14)}=16.93, P<0.05\right)$.

\section{DISCUSSION}

These experiments demonstrated that intermittent access to sucrose exerted a long-term effect on dopamine-dependent learning mechanisms, specifically, an attenuation of the blocking effect. They also showed this effect involves an alteration in $\mathrm{D}_{2}$ receptors as systemic administration of the $\mathrm{D}_{2}$ agonist QUI restored blocking in rats that had received sucrose exposures. Chronic lateral-ventricular ghrelin infusions also attenuated the blocking effect, suggesting that alterations in ghrelin signaling could be involved in the effect of sucrose intake on the dopaminergic system. This suggestion was supported by the finding that rats with a history of either sucrose or ghrelin treatment exhibited increased locomotor activity following amphetamine challenge, indicating that both treatments altered dopaminergic circuits in the midbrain (Kalivas and Stewart, 1991). Taken together, these data suggest that intermittent exposure to sucrose disrupts $D_{2}$ receptor signaling, resulting in the formation of aberrant food-cue associations. We propose that a disruption of ghrelin signaling acts as an intermediary between sucrose exposure and dopaminergic change; specifically, the expectation of highly palatable sucrose at the same time each day produces large bursts of ghrelin activity outside their normal nocturnal feeding cycles, leading to changes in dopaminergic activity.

The deficit in blocking suggests that ISI impaired the error-correction mechanisms that regulate associative formation. That is, learning in rats with a history of ISI was not controlled by the usual dopaminergic-dependent mechanism whereby a teaching signal is elicited by an unexpected outcome, and which wanes when the outcome is fully predicted, restricting learning to good predictors of the outcome (Schultz et al, 1997). Additional evidence for a specific deficit in prediction error is supported by the finding that rats given ISI could use the two auditory stimuli, B and $\mathrm{D}$, to predict two distinct outcomes to bias a choice between actions earning that same outcome (ie, the Pavlovian-toinstrumental transfer effect, see Supplementary Figure 1). This demonstrates that the failure to detect blocking is not due to generalization between stimuli or a deficit in directing responding towards a cue, which has previously signaled a motivationally significant outcome. However, the mechanistic basis of the effect of ISI on prediction error is unclear. For example, ISI may have impacted subsequent learning by altering the firing patterns of midbrain dopamine neurons that generate the prediction-error signal. Alternatively, ISI may have impacted at the level of dopaminergic receptors that receive the prediction-error signal and translate the signal into changes in the activity of other neurons.

A disruption of the teaching signal elicited by prediction error could contribute to maladaptive feeding behaviors exhibited by people who develop obesity. Stimuli in the environment have a significant role in promoting feeding and can elicit feeding even in satiated rats (Holland and Petrovich, 2005; Volkow et al, 2011). The blocking effect examined here is an example of how dopaminergic systems regulate, which environmental stimuli are learnt about. These systems have evolved to direct learning towards cues that are better predictors of a motivationally significant outcome to facilitate adaptive behavior, at the expense of cues that are worse predictors (Mackintosh, 1975; Rescorla and Wagner, 1972; Schultz et al, 1997). The present results demonstrate that a history of intermittent access to a hedonic substance produces aberrant food-cue associations, eliciting approach to food cues that would usually be blocked by prior learning. Essentially, normally ineffective cues came to control food approach, implying that the history of sucrose exposure essentially increases the number of environmental cues that can elicit an approach to food. In the development of obesity, intermittent access to hedonic foods, such as those that are rich in sugar, may disrupt the control over feeding behavior by normally predictive environmental, social, and temporal cues because of the impact of such foods on the dopaminergic-based error-correction mechanisms. 
We also found that the blocking deficit induced by a history of sucrose was removed by systemic injection of a $\mathrm{D}_{2}$ agonist. This is important in the context of human research demonstrating that body mass index is negatively correlated with $\mathrm{D}_{2}$ availability (Wang et al, 2001). This correlation led to suggestions that a blunted dopaminergic response could increase feeding behaviors. However, here we demonstrated that this does not necessarily result in a blunted response to reward per se; rather, that intake of palatable foods disrupts the dopaminergic processes, which regulate the formation of associations between cues and food. This suggestion has implications for therapeutic interventions to curb maladaptive feeding patterns in people with obesity, where pharmacotherapy targeting $\mathrm{D}_{2}$ receptors is not sufficient to produce changes in eating (Blum et al, 2014). Rather, such therapy should be combined with psychological interventions aimed at correcting existing aberrant food-cue associations.

It is not immediately clear how a change in dopamine receptors leads to a disruption of a prediction-error mechanism. That is, this signal originates in dopaminergic neurons in the VTA (Schultz et al, 1997), but the reported changes resulting from ISI occur in $\mathrm{D}_{1}$ and $\mathrm{D}_{2}$ receptors in the striatum (Avena et al, 2008; Furlong et al, 2014). Recent suggestions are that $D_{1}$ and $D_{2}$ receptors facilitate the translation of the dopaminergic prediction-error signal into learned associations at the cellular level. Specifically, although $\mathrm{D}_{2}$ receptors are at high occupancy at low tonic levels of dopamine, $\mathrm{D}_{1}$ receptors increase occupancy only at high concentrations like those supported by phasic bursts of activity resulting from prediction error signals in the VTA (Richfield et al, 1989; Schultz, 2007; Dreyer et al, 2010). Moreover, there is evidence that $D_{1}$ and $D_{2}$ receptors mediate late long-term potentiation and long-term depression, respectively, in striatal pathways to influence long-term synaptic plasticity (for reviews see Calabresi et al (2007) and Lisman et al (2011)). This evidence has led to the proposal that high levels of $\mathrm{D}_{2}$ occupancy at tonic low levels of dopamine inhibit learning about cues that do not signal significant dopaminergic change and that it is only high levels of dopamine which activate $D_{1}$ receptors to facilitate the development of learned associations when a prediction error is present (Schultz, 2007, 2013). According to this proposal, a decrease in $\mathrm{D}_{2}$ occupancy at tonic levels of dopamine produced by sucrose treatment (a probable consequence of changes in $\mathrm{D}_{2}$ expression and availability; Johnson and Kenny, 2010) reduced the inhibition that suppresses learning about non-predictive cues (ie, the blocked cue B), whereas the artificial increase in dopamine tone by administration of the $\mathrm{D}_{2}$ agonist QUI restores this inhibition. Although this interpretation is speculative, it is consistent with the finding that QUI restored blocking in animals given sucrose treatment by reducing learning about the blocked stimulus B rather than increasing learning about D. In the same vein, reduced levels of learning about D in experiment 1 (although not replicated in experiment 2 ) could also point to changes in $D_{1}$ receptor occupancy at high levels of dopamine, which may have slowed learning about the compound CD across the short number of sessions used.

Other research assessing the impact of intermittent intake of palatable foods has attributed long-term changes in behaviour to an imbalance in $\mathrm{D}_{1}$ and $\mathrm{D}_{2}$ dopamine receptors in striatal pathways (Volkow et al 2011; Furlong et al, 2014;
Reichelt et al, 2015). For example, Furlong et al (2014) demonstrated that rats given intermittent access to sweetened condensed milk subsequently acquired habits more rapidly than control rats, evidenced by the continued performance of an instrumental response in spite of devaluation of its food associate. Further, they demonstrated that administration of a $\mathrm{D}_{1}$ antagonist into the dorsolateral striatum reversed this effect. Recently, ISI has also been found to produce a subsequent deficit in a rodent model of the Stroop task; a task that requires top-down control over goal-directed behaviors (Reichelt et al, 2015), known to be dependent on fronto-striatal systems and $D_{1}$ receptors (Haddon and Killcross, 2011). The present data demonstrate that this imbalance may also impact on the error-correction mechanisms that regulate Pavlovian associations. Intermittent access to palatable substances, therefore, appears to result in enduring changes (up to 1 month after exposure in the present experiments) in the balance between $D_{1}$ and $D_{2}$ receptors, which has diverse consequences for behaviour.

The finding that repeated ghrelin infusions changed dopaminergic-based learning processes suggests that disruption of ghrelin signaling underlies the dopaminergic dysfunction resulting from ISI. Although a direct link between sucrose intake and changes in ghrelin signaling is lacking, the finding that both sucrose and ghrelin treatment enhanced amphetamine-induced locomotor activity implicates a similar change in dopaminergic receptors in the VTA-NaCC pathway as a consequence of these treatments (Kalivas and Stewart, 1991). It is now well established that peripheral ghrelin enters the brain to influence dopaminergic neurons, but the specific nature of this influence on dopamine functioning in producing the present results is unclear. For example, the expectation of palatable foods at the same time each day may elicit an exaggerated ghrelin response and/or the repeated occurrence of this response outside of normal feeding patterns produced the effect of ISI on blocking. Direct measurement of ghrelin during ISI would be useful in assessing these suggestions and providing more direct evidence for the proposed role of ghrelin in mediating the effects of ISI on subsequent learning. Given findings that chronic infusions of ghrelin produce a decrease in dopamine receptor expression (Skibicka et al, 2012), in contrast to the increase in dopaminergic firing found with acute infusions (Cone et al, 2014), persistent increases in endogenous ghrelin via either of the mechanisms described above may cause long-term reduction in dopamine receptor expression as a compensatory reaction to the higher levels of dopamine. What is clear is that ghrelin has the capacity to result in longterm changes to midbrain dopaminergic pathways, with implications for the potential role of ghrelin in obesity treatments.

Finally, the finding that ISI altered the subsequent locomotor response to amphetamine suggests a potential overlap in the mechanisms underlying palatable foods and psychoactive drugs (where chronic administration of drugs of abuse also produces enhanced locomotor activity with amphetamine challenge; Kalivas and Stewart, 1991); an overlap which has contributed to the view that the eating elicited by hedonically attractive food is a form of addiction (Volkow and Wise, 2005). Moreover, the finding that ghrelin infusions also altered the response to amphetamine suggests that ghrelin also has a role in addictive processes. This is 
consistent with recent findings that ghrelin antagonism decreases cocaine-induced locomotor sensitization and central ghrelin infusions increases the potentiation of ethanol consumption in rats given cocaine (Clifford et al, 2012; Cepko et al, 2014). It has been pointed out that psychoactive drugs are typically taken intermittently, similar to the schedules used in animal models of addiction (Jentsch et al, 2002; Furlong et al, 2014). As the present experiments suggest, the intermittency of intake may be critical in predicting whether such intake results in long-term changes in dopaminergic and ghrelin signaling. Future experiments investigating schedules under which psychoactive drugs may cause long-term changes to dopaminergic systems would elucidate these issues.

\section{FUNDING AND DISCLOSURE}

This research was supported by a project grant from the National Health and Medical Research Council of Australia to MJM and RFW. The authors declare no conflict of interest.

\section{REFERENCES}

Abizaid A, Liu ZW, Andrews ZB, Shanabrough M, Borok E, Elsworth JD et al (2006). Ghrelin modulates the activity and synaptic input organisation of midbrain dopamine neurons while promoting appetite. J Clin Invest 116: 3229-3239.

Andrews N, Legg E, Lisak D, Issop Y, Richardson D, Harper S et al (2012). Spontaneous burrowing behavior in the rat is reduced by peripheral nerve injury or inflammation associated pain. Eur $J$ Pain 16: 485-495.

Avena NM, Rada P, Hoebel BG (2008). Evidence for sugar addiction: behavioral and neurochemical effects of intermittent, excessive sugar intake. Neurosci Biobehav Rev 32: 20-39.

Baxter MG, Gallagher M, Holland PC (1999). Blocking can occur without losses in attention in rats with selective removal of hippocampal cholinergic input. Behav Neuroscience 113: 881-890.

Blum K, Thanos PK, Gold MS (2014). Dopamine and glucose, obesity, and reward deficiency syndrome. Front Psychol 5: 919.

Calabresi P, Picconi B, Tozzi A, Di Fillippo M (2007). Dopaminemediated regulation of corticostriatal synaptic plasticity. Trends Neurosci 30: 211-219.

Cepko LC, Selva JA, Merfeld EB, Fimmel AI, Goldberg SA, Currie PJ (2014). Ghrelin alters the stimulatory effect of cocaine on ethanol intake following mesolimbic or systemic administration. Neuropharmacology 85: 224-231.

Clifford PS, Rodriguez J, Schul D, Hughes S, Kniffen T, Hart N et al (2012). Attenuation of cocaine-induced locomotor sensitisation in rats sustaining genetic or pharmacologic antagonism of ghrelin receptors. Addict Biol 17: 956-963.

Cone JJ, McCutcheon JE, Roitman MF (2014). Ghrelin acts as an interface between physiological state and phasic dopamine signalling. J Neurosci 34: 4905-4913.

Day JJ, Roitman MF, Wightman RM, Carelli RM (2007). Associative learning mediates dynamic shifts in dopamine signalling in the nucleus accumbens. Nat Neurosci 10: 1020-1028.

Dreyer JK, Herrik KF, Berg RW, Hounsgaard JD (2010). Influence of phasic and tonic dopamine release on receptor activation. J Neurosci 30: 14273-14283.

Egecioglu E, Jerlhag E, Salomé N, Skibicka KP, Haage D, Bohlooly-Y M et al (2010). Ghrelin increases intake of rewarding food in rodents. Addict Biol 15: 304-311.

Furlong TM, Jayaweera HK, Balleine BW, Corbit LH (2014). Binge-like consumption of a palatable food accelerates habitual control of behavior and is dependent on activation of the dorsolateral striatum. J Neurosci 34: 5012-5022.

Haddon JE, Killcross S (2011). Rat prefrontal dopamine and cognitive control: impaired and enhanced conflict performance. Behav Neurosci 125: 344-349.

Holland PC, Petrovich GD (2005). A neural systems analysis of the potentiation of feeding by conditioned stimuli. Physiol Behav 86: 747-761.

Iordanova MD, McNally GP, Westbrook RF (2006). Opioid receptors in the nucleus accumbens regulate attentional learning in the blocking paradigm. $J$ Neurosci 26: 4036-4045.

Jentsch JD, Olausson P, De La Garza R 2nd, Taylor JR (2002). Impairments of reversal learning and response perseveration after repeated, intermittent cocaine administration in monkeys. Neuropsychopharmacology 26: 183-190.

Jerlhag E (2008). Systemic administration of ghrelin induces conditioned place preference and stimulates accumbal dopamine. Addict Biol 13: 358-363.

Johnson PM, Kenny PJ (2010). Dopamine D2 receptors in addiction-like reward dysfunction and compulsive eating in obese rats. Nat Neurosci 13: 635-642.

Kalivas PW, Stewart J (1991). Dopamine transmission in the initiation and expression of drug- and stress-induced sensitization of motor activity. Brain Res Rev 16: 223-244.

Kendig MD, Boakes RA, Rooney KB, Corbit LH (2013). Chronic restricted access to a $10 \%$ sucrose solution in adolescent and young adult rats impairs spatial memory and alters sensitivity to outcome devaluation. Physiol Behav 120: 164-172.

Knox D, George SA, Fitzpatrick CJ, Rabinak CA, Maren S, Liberzon I (2012). Single prolonged stress disrupts retention of extinguished fear in rats. Learn Mem 19: 43-49.

Le Sauter J, Hoque N, Weintraubb M, Pfaff DW, Silver R (2009). Stomach ghrelin-secreting cells as food-entrainable circadian clock. Proc Natl Acad Sci USA 106: 13582-13587.

Lisman J, Grace AA, Duzel E (2011). A neoHebbian framework for episodic memory; a role for dopamine-dependent late LTP. Trends Neurosci 34: 536-547.

Mackintosh NJ (1975). A theory of attention: variations in the associability of stimuli with reinforcement. Psychol Rev 82: 276-298.

Nelson AJ, Killcross S (2013). Accelerated habit formation following amphetamine exposure is reversed by D2, but enhanced by D2, receptor antagonists. Front Neurosci 7: 76.

Paxinos G, Watson C (1998). The Rat Brain in Stereotaxic Coordinates. Academic press: San Diego, CA, USA.

Reichelt AC, Killcross S, Hambly LD, Morris MJ, Westbrook RF (2015). Impact of adolescent sucrose access on cognitive control, recognition memory, and parvalbumin immunoreactivity. Learn Mem 22: 215-224.

Rescorla RA, Wagner AR (1972). A theory of Pavlovian conditioning: variations in the effectiveness of reinforcement and nonreinforcement. In: Black AH, Prokasy WF (eds), Classical Conditioning: Vol. 2. Current Research and Theory. AppletonCentury-Crofts: New York, USA, pp 64-99.

Rhodes SE, Killcross S (2004). Lesions of the rat infralimbic cortex enhance recovery and reinstatement of an appetitive Pavlovian response. Learn Mem 11: 611-616.

Richfield EK, Penney JB, Young AB (1989). Anatomical and affinity state comparisons between dopamine D1 and D2 receptors in the rat central nervous system. Neuroscience 30: 767.

Savage SW, Zald DH, Cowan RL, Volkow ND, Marks-Shulman PA, Kessler RM et al (2014). Regulation of novelty seeking by midbrain dopamine D2/D3 signalling and ghrelin is altered in obesity. Obesity 22: 1452-1457.

Schellekens H, Dinan TG, Cryan JF (2013). Ghrelin at the interface of obesity and reward. Vitam Horm 91: 285-323.

Schultz W (2007). Multiple dopamine functions at different time courses. Annu Rev Neurosci 30: 259-288. 
Schultz W (2013). Updating dopamine reward signals. Curr Opin Neurobiol 23: 229-238.

Schultz W, Dayan P, Montague PR (1997). A neural substrate of prediction and reward. Science 275: 1593-1599.

Sharpe M, Killcross S (2014). The prelimbic cortex contributes to the down-regulation of attention towards redundant cues. Cereb Cortex 24: 1066-1074.

Sharpe M, Killcross S (2015). The prelimbic cortex uses contextual cues to modulate responding towards predictive cues during fear renewal. Neurobiol Learn Mem 118: 20-29.

Skibicka KP, Hansson C, Egecioglu E, Dickson SL (2012). Role of ghrelin in food reward: impact of ghrelin on sucrose self- administration and mesolimbic dopamine and acetylcholine receptor gene expression. Addict Biol 17: 95-107.

Van de Giessen E, Celik F, Schweitzer DH, van den Brink W, Booij J (2014). Dopamine D2/D3 receptor availability and amphetamineinduced dopamine release in obesity. J Psychopharmacol 28: 866-873.

Volkow ND, Wang GJ, Baler RD (2011). Reward, dopamine and the control of food intake: implications for obesity. Trends Cogn Sci 15: 37-46.

Volkow ND, Wise RA (2005). How can drug addiction help us understand obesity? Nat Neurosci 8: 550-560.

Wang GJ, Volkow ND, Logan J, Pappas NR, Wong CT, Zhu W et al (2001). Brain dopamine and obesity. Lancet 357: 354-357.

Supplementary Information accompanies the paper on the Neuropsychopharmacology website (http://www.nature.com/npp) 